\title{
Virtual care in the ED: a game changer for the future of our specialty?
}

\author{
Kendall $\mathrm{Ho}^{1,2}$ (1)
}

Received: 8 November 2020 / Accepted: 28 November 2020

(c) Canadian Association of Emergency Physicians (CAEP)/ Association Canadienne de Médecine d'Urgence (ACMU) 2020

In this issue of CJEM, the article by Reid et al. [1] on the implementation of virtual care the use of information technologies to deliver health services, also known as telehealth, in a pediatric emergency department is an important trend of our discipline to take note. This approach is a significant game changer in how we deliver tele-triage and remote treatment as one important facet of emergency medicine to serve our patient population.

A recent article spoke to a progressive rise in interest in telehealth in three categories of services: clinician to clinician, clinician to patient, and patient to mobile health technologies [2]. This article further predicted five key trends in fueling telehealth adoption: innovation in consumer technology market, advancement in electronic health records, shortage in health professional workforce, health system reorganization and financing, and growth in consumerism [2].

COVID has propelled telehealth from early adoption into the mainstream of our health care systems in Canada and abroad. A recent report in US, based on several surveys during COVID, suggested that consumer adoption of telehealth increased from $11 \%$ in 2019 to $46 \%$ to replace canceled healthcare visits, and providers increased the use of telehealth for patient care by $50-175$ times [3]. Also, the Canadian Medical Association conducted a national poll in May 2020, showing that almost half of all Canadians have accessed physicians via virtual care [4]. Those who experienced virtual care expressed a $91 \%$ satisfaction rate, and $46 \%$ would prefer virtual care as the first point of contact with their doctors [4].

Emergency medicine is also increasingly interested in the adoption of telehealth into our discipline. For example, even before COVID, Society for Academic Emergency

Kendall Ho

kendall.ho@ubc.ca

1 Department of Emergency Medicine, Faculty of Medicine, University of British Columbia, Vancouver, Canada

2 Cloud Innovation Centre for Community Health and Wellbeing, University of British Columbia, Vancouver, Canada
Medicine focused on telehealth as the topic of its Consensus Conference in 2020, [5] a choice that portended the rise of this practice upon COVID's arrival. Canadian emergency medicine colleagues also call for consideration of incorporating telehealth into our practice in light of COVID surge, so as to enable physical distancing and reducing physical interactions between patients and health professionals to reduce transmission risk in the ED [6]. Having examples of telehealth use to demonstrate how care in the ED can be improved is necessary and instructive to translate concepts into practice.

In a timely and useful way, the Reid et al. paper describes a prospective cohort study to illuminate on how a tertiary pediatric emergency department in Canada can implement virtual care to serve patients and their parents during this COVID time, using a secure encrypted video platform to interview patients remotely [1]. Overall, a vast majority of patients were appropriately treated, and the service was well liked by parents and patients overall. The in-person visit burden to the ED was also reduced. While this model is attractive and this study demonstrated its feasibility, further scrutiny regarding safety of this service is needed, so that the use of virtual care does not inadvertently lead to delay in emergency care for patients that need to present to ED after virtual encounters. Also, determining how best to establish the clinical guidelines to stratify patients to virtual care, and implementing this service in other EDs for validation would be highly useful.

An increasing number of emergency virtual care models are being described in the literature, such as pre-hospital care, medical trainee oversight, or specialty consultations [7, 8]. These new pathways of care, and emerging virtual care models in the future, need to be validated for their utility and safety for patient care. Reid et al illustrates how to conduct a timely feasibility study to commence the validation journey to integrate a novel virtual care model into mainstream emergency medicine practice. Sharing of proven virtual care protocols amongst EDs would further accelerate its adoption into our specialty. 
While COVID-19 has brought havoc to our society and our health system, it also marks the dawn of mainstreaming virtual care in our specialty. This technology-enabled approach will almost certainly be entrenched into our health service delivery even after COVID-19 is brought under control. Further evaluation of the various ways virtual care can be introduced into ED, and how they can be brought to scale so as to bring widespread benefits to our communities and their members will truly be transformative and an aspirational goal worthy of pursuit.

\section{References}

1. Reid J, et al. Virtual care in the pediatric emergency department. A new way of doing business? CJEM. 2020. https://doi.org/10.1007/ s43678-020-00048-w.

2. Tuckson RV, Edmunds M, Hodgkins ML. Telehealth. N Engl J Med. 2017;377:1585-92. https://doi.org/10.1056/NEJMsr150323.

3. McKinsey \& Company. Telehealth: a quarter-trillion-dollar postCOVID-19 reality. 2020. https://www.mckinsey.com/industries/ healthcare-systems-and-services/our-insights/telehealth-a-quart er-trillion-dollar-post-covid-19-reality. Accessed 3 Nov 2020.

4. Canadian Medical Association. Virtual care is real care: national poll shows Canadians are overwhelming satisfied with virtual health care. 2020. https://www.cma.ca/news/virtual-care-realcare-national-poll-shows-canadians-are-overwhelmingly-satis fied-virtual. Accessed 3 Nov 2020.

5. SAEM Consensus Conference. Telehealth and Emergency Medicine: a consensus conference to map the intersection of Emergency Medicine and Telehealth. 2020. https://www.saem.org/ annual-meeting/future-and-past-meetings/saem-consensus-confe rence-2020. Accessed 3 Nov 2020.

6. Masood S, Chartier LB. Enhanced virtual care in the emergency department as a strategy to safely manage the COVID-19 surge. Letter to the Editor. CJEM. 2020. https://doi.org/10.1017/ cem.2020.429.

7. Russi CS, Heaton HA, Demaerschalk BM. Emergency medicine telehealth for COVID-19. Mayo ClinProc. 2020;95(10):2065-8.

8. Hamm JM, Greene C, Sweeney M, Mohammadie S, Thompson LB, Wallace E, Schrading W. Telemedicine in the emergency department in the era of COVID-19: front-line experiences from 2 institutions. JACEP. 2020. https://doi.org/10.1002/emp2.12204. 